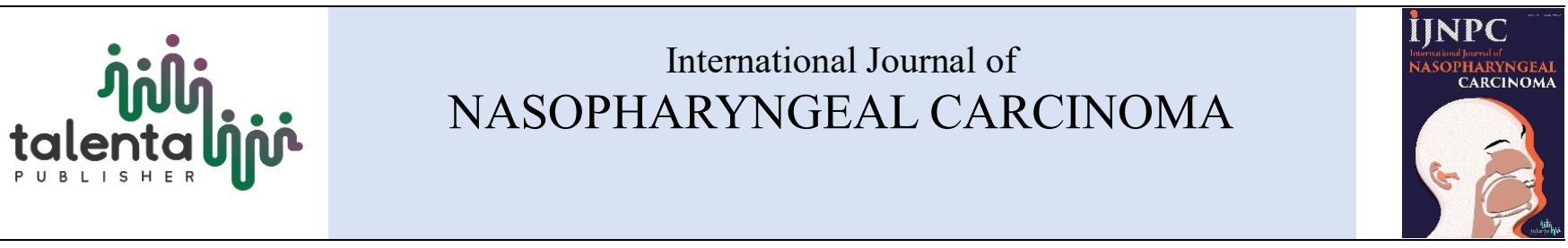

\title{
EARLY-STAGE NASOPHARYNGEAL CARCINOMA: A CASE REPORT
}

\author{
Izry Naomi Tobing ${ }^{*}$ \\ Otorhinolaryngology Head and Neck Surgery Departement, Faculty of Medicine, Universitas Sumatera Utara, Indonesia
}

\section{Abstract}

Introduction: NPC is a highly prevalent malignant disease in endemic regions such as Southeast Asia. Until now, most NPC patients have bben diagnosed in advanced stages. Early-stage NPC is difficult to diagnose clinically. The study present a case of early-stage NPC with the symptoms of progressive ringing in the ear. Case report: A 56 years old male presented with two months history of progressive unilateral ringing in the ear. The patient had been previously diagnosed and treated for Eustachian tube dysfunction. Endoscopic examination of nasopharynx showed a mass in the left fossae of Rosenmüller. CT scan depicted soft tissue thickening on the left side of the nasopharynx. Nasopharyngeal histopathology revealed a non-keratinizing squamous cell carcinoma. The patient underwent radiotherapy and up to date, no complaints have been encountered.

Conclusion: Early detection of NPC will improve patient's prognosis.

\section{Article Info}

Keywords:

Nasopharyngeal carcinoma, early-stage, ear-related symptoms, early detection

\section{*Corresponding author:}

Address: Otorhinolaryngology Head and Neck Surgery Department, Faculty of Medicine, Universitas Sumatera Utara, Indonesia

e-mail: izrynaomi@gmail.com

\section{CASE REPORT}

In August 8, 2013, a 56 years old Bataknese male presented to the outpatient clinic of ENT Department of General Hospital of H. Adam Malik, Medan with two months history of progressive unilateral ringing in the ear. There were associated hearing impairment and feeling of fullness in the ipsilateral ear, but no otalgia, no ear discharge, and no balance disorders. Nasal blockage and post nasal drip were experienced sometimes, but there were no nasal discharge and epistaxis. Throat problems and neuro-ophthalmic symptoms were denied. There were no complains of neck swelling. A history suggesting exposure to carcinogens was denied. The patient has been previously diagnosed and treated for Eustachian tube dysfunction in another hospital, but there was no improvement.

Routine ENT examination revealed a dull left tympanic membrane with loss of light reflex. No abnormalities were found in the contralateral ear. Anterior nasal and oropharyngeal examinations revealed normal findings. However, endoscopic examination of nasopharynx showed a mass in the left fossae of Rosenmüller which can be seen through the left nasal cavity.

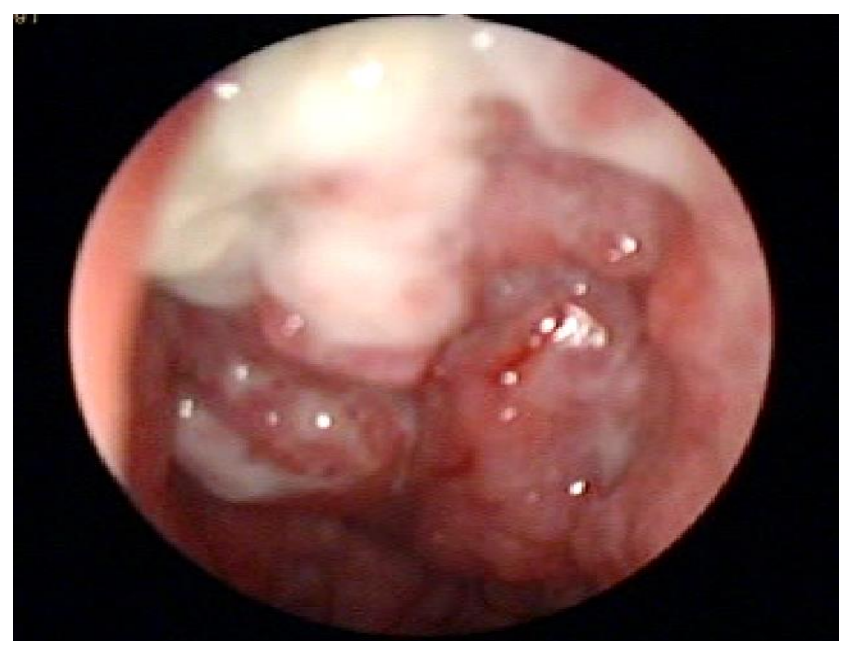

Picture 1. An endoscopic feature of nasopharyngeal carcinoma in the patient
A nasopharyngeal biopsy was performed. The histopathology result revealed a non-keratinizing squamous cell carcinoma. Computerized axial tomogram (CT-scan) of the nasopharynx with contrast was ordered to determine tumor extensions. The CT scan revealed soft tissue thickening on the left side of the nasopharynx, the left fat plane was partly obliterated and no lymph node enlargement. Thorax x-ray, abdominal ultrasonography, and bone scan showed no further metastases.

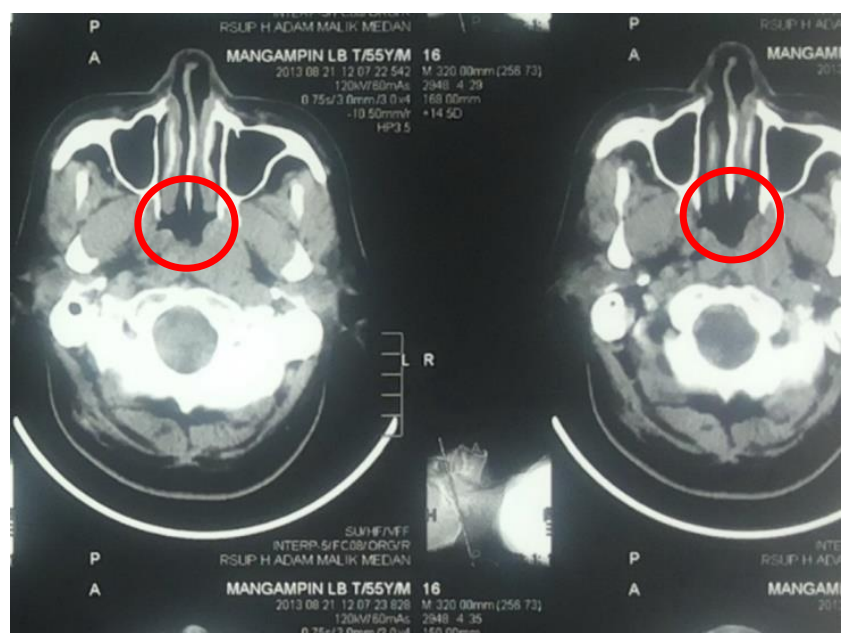

Picture 2. Computed tomography of the nasopharynx.

The patient was diagnosed with nasopharyngeal carcinoma stage I (T1N0M0) and prepared for radiotherapy in September 2013. The patient underwent radiotherapy for 35 fractions for a total dose of $70 \mathrm{~Gy}$. A follow up of the patient a month after the treatment indicates no significant symptoms. No mass to be found in the endoscopic examination despite the surface nasopharyngeal mucosal irregularity. 


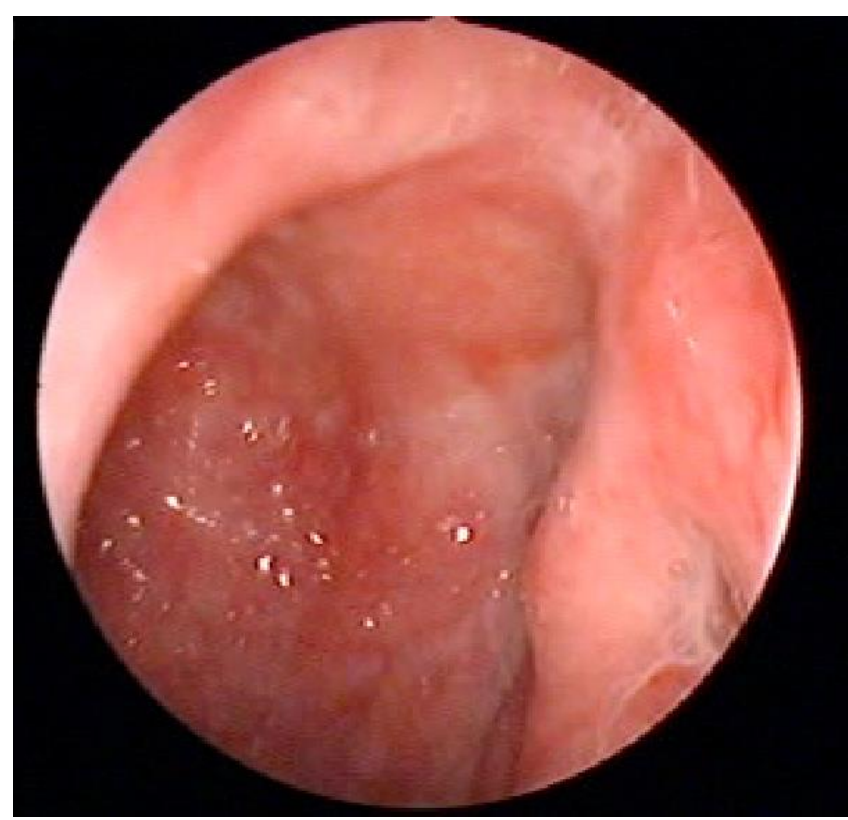

Picture 3. An endoscopic feature of the patient's nasopharynx a month after the treatment.

\section{DISCUSSION}

Nasopharyngeal carcinoma (NPC) is one of the head and neck squamous cell carcinoma that is very common in the endemic geographical regions such as Southern China, Southeast Asia, Japan, and the Middle East/North Africa [1]. According to the International Agency for Research on Cancer report in 2008, nasopharyngeal cancer accounts for more than $80 \%$ in these regions compared to those reported in Europe that is only $5 \%$. To be more specific, $71 \%$ of new nasopharyngeal cancer cases are reported in East and Southeast Asia, and 29\% are in South and Central Asia and North and East Africa [2].

Consumption of salted fish that is related to the carcinogenic compound, Nitrosamine, is one of the etiologic factors most frequently mentioned. The consumption of salted fish at childhood contributes to the increased incidence. The Epstein-Barr virus (EBV) genome is frequently detected in the biopsy specimens of NPC. Therefore it has also been considered to play an oncogenic role in this tumor [3]. In this patient, history suggesting of exposure to carcinogens were denied.

As for the infection of EBV, while it is very common and affecting over $95 \%$ of the global adult population, largely asymptomatic and life-long, it is the undifferentiated form of (NPC) that shows the most consistent worldwide association with EBV [4]. This patient's histopathology result revealed a non-keratinizing squamous cell carcinoma.

Symptoms of NPC frequently present with one or more of 4 groups of symptoms. They are consist of: 1 . Nasal symptoms such as epistaxis, nasal obstruction and discharge (these symptoms are related to the presence of tumor mass in the nasopharynx; 2. Otologic symptoms such as deafness and tinnitus, related to the Eustachian tube dysfunction caused by the lateroposterior extension of the tumor to the paranasopharyngeal space; 3. Cranial nerve palsies, commonly 5th and 6th cranial nerves, which is associated with the superior extension of the tumor leading to skull base erosion (the patient might experience a headache, diplopia, facial pain, and numbness); and 4. Neck masses, usually appear in the upper neck [5].

Patients with an early stage of the disease may present with ear-related complaints. A small tumor in the fossa of Rosenmüller of the nasopharynx may cause Eustachian tube dysfunction. The tumor may spread outward via the mucosa and sub-mucosa, or along the muscle bundles within the fibrofatty tissue planes or the neuro-vascular planes. Tinnitus as an isolated symptom in the absence of other features relating to the ear is highly unlikely. If present, it is normally a result of the Eustachian tube, middle ear or auditory nerve involvement with the resulting associated aural manifestations as well [6]. Although a recent study showed that most Indonesian NPC patients had recognized the earliest sign of NPC which is unilateral ear problem $(60.6 \%)$, data indicated that neither patients nor doctors gave this condition attention until cervical lymph node enlargement, a sign of late-stage NPC was detected [7].

Only $10 \%$ of NPC patients are likely to be diagnosed early at stage I [8]. Early-stage NPC is difficult to diagnose clinically because of its hidden location in the nasopharynx [9]. Furthermore, the presentations of NPC are variable but may also remain asymptomatic for a long time, making it harder to detect the occult site of presentation. Moreover, sometimes patients consult doctors of different specialties who have little experience in managing nasopharyngeal carcinoma [10].

In this case, the patient had been experiencing two-months of progressive unilateral tinnitus. There was associated hearing impairment and feeling of fullness in the ipsilateral ear. Also, the patient has been treated for Eustachian tube dysfunction, but there was no improvement. A thorough nasoendoscopic examination and awareness of NPC early signs and symptoms had made it possible for us to detect an early-stage NPC.

WHO classification of NPC in 2005 divided NPC into three major types. They are keratinizing squamous cell carcinoma, non-keratinizing carcinoma, and basaloid squamous cell carcinoma. Histopathology result, in this case, revealed that the tumor was a non-keratinizing squamous cell carcinoma. It comprises over $95 \%$ of NPC in high incidence areas and approximately $75-87 \%$ of NPC in low incidence areas. These types of tumors are generally more radiosensitive compare to squamous cell carcinoma and have stronger relationships with EBV [11]

Computed tomography (CT) has long been utilized for staging NPC, especially to detect skull base tumor involvement with lytic or sclerotic lesions. However, now, in some centers, MRI has replaced the use of CT for primary and nodal staging. MRI is considered to be an accurate examination to diagnose NPC. Subclinical NPCs that are missed at endoscopy can be detected by MRI. NPCs in MRI usually present with intermediate signal intensity on T2-weighted images and low signal intensity on T1-weighted images. Most NPCs arise in the posterolateral recess of the pharyngeal wall (Rosenmüller fossa) (82\%), 12\% arise in the midline and $6-10 \%$ of patients showed normal nasopharyngeal mucosa appearance at endoscopy [12]. However, CT is still needed in radiotherapy planning.

When suspicion of early-stage NPC arises, MRI can be an option if endoscopic examination does not reveal the expected signs. In this case, the CT scan revealed soft tissue thickening on the left side of the nasopharynx and the left fat plane was partly obliterated. No further examinations are needed.

NPC is highly radiosensitive and radiotherapy is the mainstay of treatment for all stages of NPC without distant metastases with a 5-year overall survival of $75-90 \%[5,12,13]$. For T1, N0 patients, radiotherapy alone is given with a radical dose of 66-70 Gy daily Monday-Friday in 6-7 weeks [14].

The patient underwent radiotherapy for 35 fractions for a total dose of $70 \mathrm{~Gy}$. The result is satisfactory and the patient has no complaints until this report was made. Important prognostic factors for survival in NPC are the stage, WHO type, and age at diagnosis [15]. Therefore, this patient is expected to have good prognosis.

\section{CONCLUSION}

A thorough examination and awareness of NPC's early signs and symptoms will made it possible for clinicians to detect an early-stage NPC. Stage, WHO type and age at diagnosis are important prognostic factors for survival for NPC patients.

\section{REFERENCE}

[1] Tabuchi K, Nakayama M, Nishimura B, Hayashi K, Hara A. Early Detection of Nasopharyngeal Carcinoma. International Journal of Otolaryngology. 2011;2011.

[2] Mahdavifar N, Ghoncheh M, Mohammadian-Hafshejani A, Khosravi $\mathrm{B}$, Salehiniya H. Epidemiology and Inequality in The Incidence and Mortality of Nasopharynx Cancer in Asia. Osong Public Health and Research Perspectives. 2016;7(6):360-72.

[3] Johnson JT, Rosen CA. Bailey's Head and Neck SurgeryOtolaryngology Review: Lippincott Williams \& Wilkins; 2014.

[4] Young LS, Dawson CW. Epstein-Barr Virus and Nasopharyngeal Narcinoma. Chinese Journal of Cancer. 2014;33(12):581.

[5] Wei WI, Kwong DL. Current Management Strategy of Nasopharyngeal Carcinoma. Clinical and Experimental Otorhinolaryngology. 2010;3(1):1. 
[6] Low WKC, Rangabashyam M. Ear-related Issues in Patients with Nasopharyngeal Carcinoma. Carcinogenesis, Diagnosis, and Molecular Targeted Treatment for Nasopharyngeal Carcinoma: InTech; 2012.

[7] Adham M, Kurniawan AN, Muhtadi AI, Roezin A, Hermani B, Gondhowiardjo S, et al. Nasopharyngeal Carcinoma in Indonesia: Epidemiology, Incidence, Signs, and Symptoms at Presentation. Chinese Journal of Cancer. 2012;31(4):185.

[8] Gleeson MJ, Clarke RC. Scott-Brown's Otorhinolaryngology: Head and Neck Surgery 7Ed: 3 Volume Set: CRC Press; 2008.

[9] Ali AHM, Shubber MRH. Modes of Nasopharyngeal Carcinoma Presentation. Int J Curr Microbiol App Sci. 2016;5(11):401-6.

[10] Li Z, Zong Y-s. Review of The Histological Classification of Nasopharyngeal Carcinoma. Journal of Nasopharyngeal Carcinoma. 2014;1(15).

[11] Razek AAKA, King A. MRI and CT of Nasopharyngeal Carcinoma. American Journal of Roentgenology. 2012;198(1):11-8.

[12] Qiu W-Z, Ke L-R, Xia W-X, Yang J, Yu Y-H, Liang H, et al. A Retrospective Study of 606 Cases of Nasopharyngeal Carcinoma with or Without Oropharyngeal Candidiasis During Radiotherapy. PloS One. 2017;12(8):e0182963.

[13] Wei K-R, Zheng R-S, Zhang S-W, Liang Z-H, Ou Z-X, Chen W-Q. Nasopharyngeal Carcinoma Incidence and Mortality in China in 2010. Chinese Journal of Cancer. 2014;33(8):381.

[14] Colevas AD, Yom SS, Pfister DG, Spencer S, Adelstein D, Adkins D, et al. NCCN Guidelines Insights: Head and Neck Cancers, Version 1.2018. Journal of the National Comprehensive Cancer Network. 2018;16(5):479-90.

[15] Arnold M, Wildeman MA, Visser O, Karim-Kos HE, Middeldorp JM, Fles R, et al. Lower Mortality from Nasopharyngeal Cancer in The Netherlands Since 1970 with Differential Incidence Trends in Histopathology. Oral Oncology. 2013;49(3):237-43. 